\title{
1 Contrasting mechanisms for hidden hearing loss: synaptopathy vs
}

\section{myelin defects}

3 Maral Budak $^{1}$, Karl Grosh ${ }^{2,3,5}$, Gabriel Corfas ${ }^{4,5^{*}}$, Michal Zochowski ${ }^{1,6^{* *}}$, Victoria Booth ${ }^{7 * \pi}$

4

$5 \quad{ }^{1}$ Biophysics Program, University of Michigan, Ann Arbor, Michigan, United States of America

$6 \quad{ }^{2}$ Department of Mechanical Engineering, University of Michigan, Ann Arbor, Michigan, United

7 States of America

$8{ }^{3}$ Department of Biomedical Engineering, University of Michigan, Ann Arbor, Michigan, United

9 States of America

$10{ }^{4}$ Department of Otolaryngology Head and Neck Surgery, University of Michigan, Ann Arbor,

11 Michigan, United States of America

$12{ }^{5}$ Kresge Hearing Research Institute, University of Michigan, Ann Arbor, Michigan, United States

13 of America

$14{ }^{6}$ Department of Physics, University of Michigan, Ann Arbor, Michigan, United States of America

$15 \quad{ }^{7}$ Departments of Mathematics \& Anesthesiology, University of Michigan, Ann Arbor, Michigan,

16 United States of America

$18{ }^{*}$ Corresponding authors

19 e-mails: vbooth@umich.edu (VB), corfas@med.umich.edu (GC), michalz@umich.edu (MZ)

21 These authors contributed equally to this work. 
Abstract

Hidden hearing loss (HHL) is an auditory neuropathy characterized by normal hearing thresholds but reduced amplitude of the sound-evoked auditory nerve compound action potential (CAP). It has been proposed that in humans HHL leads to speech discrimination and intelligibility deficits, particularly in noisy environments. Animal models originally indicated that HHL can be caused by moderate noise exposures or aging, and that loss of inner hair cell (IHC) synapses could be its cause. A recent study provided evidence that transient loss of cochlear Schwann cells also causes permanent auditory deficits in mice which have characteristics of HHL. Histological analysis of the cochlea after auditory nerve remyelination showed a permanent disruption of the myelination patterns at the heminode of type I spiral ganglion neuron (SGN) peripheral terminals,

34 suggesting that this defect could be contributing to HHL. To shed light on the mechanisms of

35 different HHL scenarios and to test their impact on type I SGN activity, we constructed a reduced

36 biophysical model for a population of SGN peripheral axons. We found that the amplitudes of 37 simulated sound-evoked SGN CAPs are lower and have greater latencies when the heminodes are 38 disorganized, i.e. they are placed at different distances from the hair cell rather than at the same 39 distance as seen in the normal cochlea. Thus, our model confirms that disruption of the position of 40 the heminode causes desynchronization of SGN spikes leading to a loss of temporal resolution and

41 reduction of the sound-evoked SGN CAP. We also simulated synaptopathy by removing high

42 threshold IHC-SGN synapses and found that the amplitude of simulated sound-evoked SGN CAPs

43 decreases while latencies remain unchanged, corresponding to what has been observed in noise

44 exposed animals. This model can be used to further study the effects of synaptopathy or 45 demyelination on auditory function. 


\section{Author summary}

Hidden hearing loss is an auditory disorder caused by noise exposure, aging or peripheral neuropathy which is estimated to affect $12-15 \%$ of the world's population. It is a 'hidden' disorder because subjects have normal hearing thresholds, i.e., the condition cannot be revealed by standard

51 audiological tests, but they report difficulties in understanding speech in noisy environments.

52 Studies on animal models suggest two possible pathogenic mechanisms for hidden hearing loss:

53 (1) loss of synapses between inner hair cells and auditory nerve fibers, and (2) disruption of

54 auditory-nerve myelin. In this study, we constructed a computational model of sound-evoked

55 auditory neuron fiber activity and auditory nerve compound action potential to understand how each one of these mechanisms affects nerve transmission. We show that disruption of auditorynerve myelin desynchronizes sound-evoked auditory neuron spiking, decreasing the amplitude and increasing the latency of the compound action potential. In addition, elongation of the initial axon segment may cause spike generation failure leading to decreased spiking probability. In contrast, the effect of synapse loss is only to decrease the probability of firing, thus reducing the compound

61 action potential amplitude without disturbing its latency. This model, which accurately represents

62 the in vivo findings, could be useful to make further predictions on the consequences of HHL and extend it to explore the impact of synaptopathy and myelinopathy on hearing.

\section{Introduction}

Hidden hearing loss (HHL) is defined as an auditory neuropathy characterized by changes

67 in neural sound-evoked output of the auditory nerve (AN) without hearing threshold elevation [1].

68 The prevalence of HHL has been estimated at $12-15 \%$ based on recent surveys where subjects with normal hearing thresholds reported difficulties in hearing, especially in noisy environments [2, 3$]$. 
70 HHL has been detected in animal models and humans by measuring the neural responses to

71 suprathreshold sound via tests, such as auditory brainstem response (ABR), a far-field response

72 measured by head-mounted electrodes, or compound action potential (CAP), a near-field response

73 measured from the round window. CAP and the first peak of ABR (ABR peak 1) represent the

74 activity of type I spiral ganglion neurons (SGNs) in response to sounds [1].

75 There is mounting evidence that HHL can be caused by noise exposure, aging or peripheral

76 myelin neuropathy [4-7]. After exposure to moderate noise, animals and humans have temporary

77 shifts in auditory thresholds but permanent decreases in amplitude of ABR peak 1 [4-7]. Kujawa

78 and Liberman (2009) showed that animals with this type of auditory pathology have a normal

79 complement of hair cells and SGNs, but present with loss of a subset of synaptic connections

80 between inner hair cells (IHCs) and SGNs. They also found that the degree of synapse loss

81 correlates with the magnitude of the decrease in suprathreshold responses, supporting the idea that

82 cochlear synaptopathy is the mechanism for noise-induced HHL [4]. Similar observations were

83 made regarding aging, i.e. HHL and synapse loss are the first signs of age-related hearing loss and

84 have the same time-course [5]. Importantly, it has been suggested that moderate noise and aging

85 primarily affect synapses associated with high threshold/low spontaneous rate SGN fibers [8].

86 Since these fibers can respond to sound in high background noise even when the others have been

87 saturated, their loss should lead to difficulties in processing speech in noisy environments [8]. Auditory processing requires proper myelination of auditory nerves [9]. Therefore, it has

89 been hypothesized that peripheral neuropathy resulting from myelin disorders may be another

90 cause of HHL. Individuals with peripheral neuropathies, such as Guillain-Barré Syndrome (GBS)

91 [10] and Charcot-Marie-Tooth (CMT) disease [11] have been reported to have perceptual

92 difficulties even when having normal auditory thresholds, indicating HHL. A recent study by Wan 
93 and Corfas (2017) showed that transient demyelination also causes HHL in mice, i.e. reduced ABR

94 peak 1 amplitude with normal ABR thresholds [6]. In that study, acute demyelination was induced

95 using genetically modified mice. This demyelination resulted in decreased ABR peak 1 amplitudes

96 and increased ABR peak 1 latency without auditory threshold elevation or IHC-SGN synapse loss.

97 Remarkably, these changes persisted even after remyelination of SGN fibers. Further investigation

98 with immunostaining demonstrated that the organization of the heminodes, the nodal structures

99 closest to the IHCs where action potentials are generated, were disrupted. These results suggested

100 that the location of SGN heminodes is critical for normal auditory responses and that their

101 disruption causes HHL.

102 In this study, we investigated the implications of these two HHL mechanisms, 103 synaptopathy and myelinopathy, on sound-evoked spike generation and timing in SGNs. For this

104 purpose, we constructed a reduced biophysical model consisting of a population of SGN fibers to

105 investigate how synapse loss or disruption of myelin organization affect spike generation and 106 transmission. Synaptopathy and myelinopathy were implemented by removing synapses and 107 varying the position of SGN heminodes, respectively. Model results show that heminode 108 disruption causes decay of the amplitude and increases the latency of sound-evoked CAPs. In 109 addition, significant elongation of the initial axon segment causes spike generation failure leading 110 to decreased spiking probability. In contrast, synaptopathy, solely decreases probability of firing, 111 subsequently decreasing CAP peak amplitude without affecting its latency. These results are 112 consistent with experimental observations $[4,6]$.

\section{Methods}

\section{SGN fiber model}


Type I SGNs are bipolar neurons with peripheral axon segments innervating IHCs and central axon segments projecting into cochlear nucleus (Fig 1A) [12]. In this study, a

118 compartmental model of peripheral axons of type I SGNs was constructed using the NEURON

119 simulator (version 7.6.2, [13]) as schematized in Figs 1B and C. For simplicity, we refer to

120 peripheral axons of type I SGNs as SGN fibers, throughout the paper. Each fiber consists of an

121 unmyelinated segment (length $\mathrm{L}_{u}$ ), a heminode (length $\mathrm{L}_{h}$ ) and 5 myelin sheaths following the

122 heminode, separated by 4 nodes. Each compartment has passive membrane properties described

123 by specific capacitance $\left(C_{m}\right)$ and specific membrane resistance $\left(R_{m}\right)$. Specific cytoplasmic

124 resistance $\left(R_{a}\right)$ between each consecutive compartment was modified to obtain the speed of the

125 action potential as $12-14 \mathrm{~m} / \mathrm{s}$ [14], based on the neural conduction velocity measurements of human

126 auditory nerve [15]. Sodium and potassium channels were inserted along the SGN fibers, except

127 the myelin sheaths, which only had passive membrane properties. The nominal conductances of

128 both channel types at the unmyelinated segment was 15 times less than the nodes and the heminode

129 [16], therefore action potential was initiated first at the heminode. The parameters for channel

130 dynamics were taken from [14] (see S1 File), the stochastic channels in [14] were converted into

131 deterministic ones for simplicity. This was done by multiplying channel density with the single

132 ion channel conductance to obtain deterministic conductance values (see Table 1 for all

133 parameters). The Nernst potentials for the ions $\mathrm{Na}^{+}\left(\mathrm{E}_{\mathrm{Na}}\right)$ and $\mathrm{K}^{+}\left(\mathrm{E}_{\mathrm{K}}\right)$ were set to 66 and $-88 \mathrm{mV}$,

134 respectively, and the resting potential ( $\left.\mathrm{E}_{\text {Rest }}\right)$ was $-78 \mathrm{mV}$ [17]. Simulations were done at $37^{\circ} \mathrm{C} . \mathrm{The}^{\mathrm{T}}$

135 differential equations were solved by fully implicit backward Euler method with time step $5 \mu \mathrm{s}$

136 implemented in the NEURON simulation environment (see S1 File). 


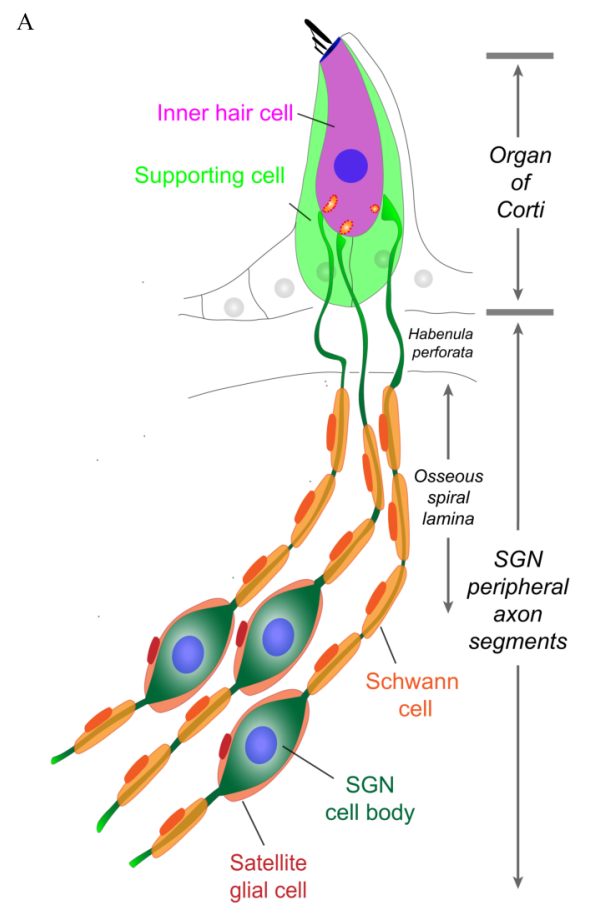

B

138 Fig 1. Diagram of model SGN fiber illustrating two mechanisms of hidden hearing loss

139 (A) Schematic illustration of type I SGNs, bipolar neurons innervating IHCs via myelinated 140 peripheral projections. (B,C) Model peripheral fibers of type I SGNs (SGN fiber) consist of an 141 unmyelinated segment at the peripheral end adjacent to the site of IHC synapses, followed by a 142 heminode and 5 myelin sheaths with 4 nodes between them. Two mechanisms of hidden hearing 143 loss are simulated: (B) synaptopathy, modeled by removing IHC-AN synapses, and (C) 144 myelinopathy, modeled by varying the lengths of the unmyelinated segment $\left(\mathrm{L}_{u}\right)$ or the heminode $145\left(\mathrm{~L}_{\mathrm{h}}\right)$. 
Table 1: Morphological, electrical and ion channel parameters of the different parts of a normal SGN fiber. Values as in [16] except for Ra and myelinated segment length which were modified for human SGN fibers.

\begin{tabular}{|c|c|c|c|c|}
\hline Parameters & Unmyelinated segment & Heminode & Myelin & Node \\
\hline Length $(\boldsymbol{\mu m})$ & 10 & 1 & 40 & 1 \\
\hline Diameter $(\boldsymbol{\mu m})$ & 1.2 & 1.2 & 2.2 & 1.2 \\
\hline $\mathbf{g}_{\mathbf{N a}}\left(\mathbf{S} / \mathbf{c m}^{\mathbf{2}}\right)$ & 0.01208 & 0.1812 & 0 & 0.1812 \\
\hline $\mathbf{g}_{\mathrm{K}}\left(\mathbf{S} / \mathbf{c m}^{\mathbf{2}}\right)$ & 0.015 & 0.225 & 0 & 0.225 \\
\hline $\mathbf{R}_{\mathbf{m}}\left(\mathbf{o h m} \mathbf{m} \mathbf{c m}^{\mathbf{2}}\right)$ & 1662 & 1662 & 1300000 & 1662 \\
\hline $\mathbf{C}\left(\boldsymbol{\mu F} / \mathbf{c m}^{\mathbf{2}}\right)$ & 0.05125 & 0.05125 & 0.0012 & 0.05125 \\
\hline $\mathbf{R}_{\mathbf{a}}(\mathbf{o h m x c m})$ & & 8291.4 & \\
\hline
\end{tabular}

151 $\mathrm{g}_{\mathrm{Na}}$, maximal sodium conductance; $\mathrm{g}_{\mathrm{K}}$, maximal potassium conductance; $\mathrm{R}_{\mathrm{m}}$, specific membrane 152 resistance; $C$, specific capacitance; $R_{a}$, specific cytoplasmic resistance.

\section{Sound representation}

Increasing sound level increases the probability of neurotransmitter release from IHCs

156 [18], therefore we defined the sound stimulus in terms of a release probability $\mathrm{p}_{\mathrm{i}}$ at each IHC-SGN

157 synapse (Fig 2A). Since type I SGNs also fire spontaneously [19], we set a release probability of

$158 \mathrm{p}_{\text {spont }}$ in the absence of sound. To simulate the activity of SGNs in response to sound stimulus as

159 described in [20], release probability was first increased sharply up to a defined peak ( $\mathrm{p}_{\text {spont }}+\mathrm{p}_{\text {peak }}$ ),

160 then allowed to decay due to adaptation to a constant level at the half-peak $\left(p_{\text {half }}=p_{\text {spont }}+\right.$

$\left.161 \frac{p_{p e a k}}{2}\right)$ until the end of the sound stimulus. Thus, the release probability function $\mathrm{p}_{\mathrm{i}}(\mathrm{t})$ was defined

162 as: 
164

$$
p_{i}(t)= \begin{cases}p_{\text {spont }} & \text { for } t<t_{\text {start }} \text { and } t>t_{\text {stop }} \\ p_{\text {spont }}+\left(\frac{p_{\text {peak }}}{0.4}\right) \times\left(e^{\left.\frac{-\left(t-t_{\text {start }}\right)}{0.8}-e^{\frac{-\left(t-t_{\text {start }}\right)}{0.3}}\right)}\right. & \text { for } t_{\text {start }}<t<t_{\text {half }} \\ p_{\text {spont }}+p_{\text {half }} & \text { for } t_{\text {half }}<t<t_{\text {stop }}\end{cases}
$$

165

166 where $t_{\text {half }}$ was the time at which the function decays to $\mathrm{p}_{\text {spont }}+\mathrm{p}_{\text {half }}$ after passing $\mathrm{p}_{\text {peak }}, \mathrm{t}_{\text {start }}$ and $\mathrm{t}_{\text {stop }}$

167 were the times when sound stimulus starts and ends, respectively. 
A

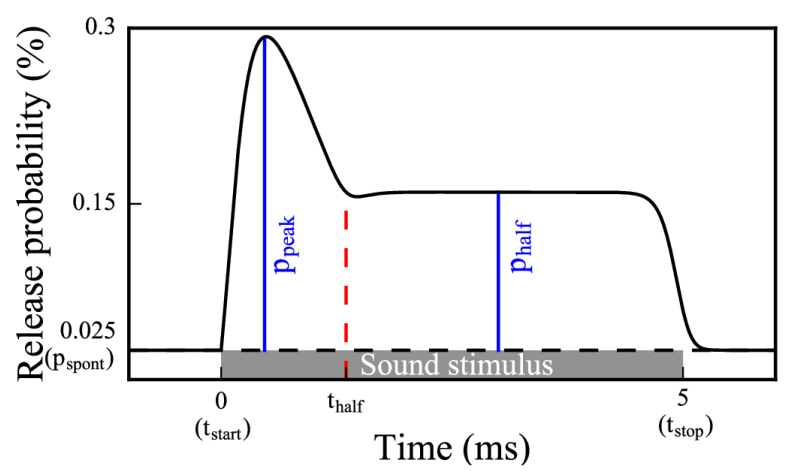

$\mathrm{C}$

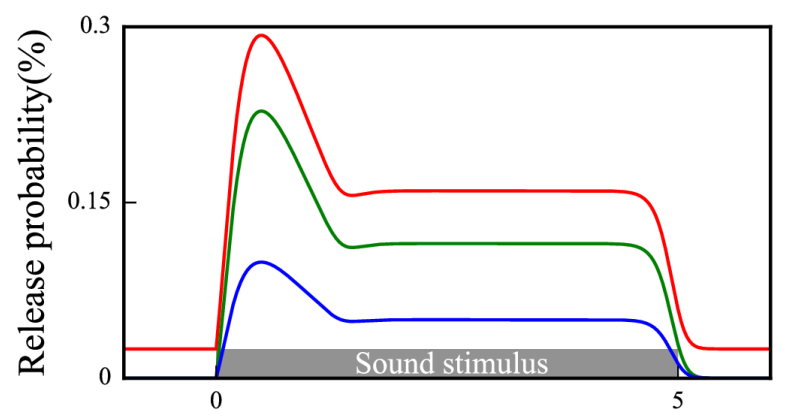

Time (ms)

$\mathrm{E}$

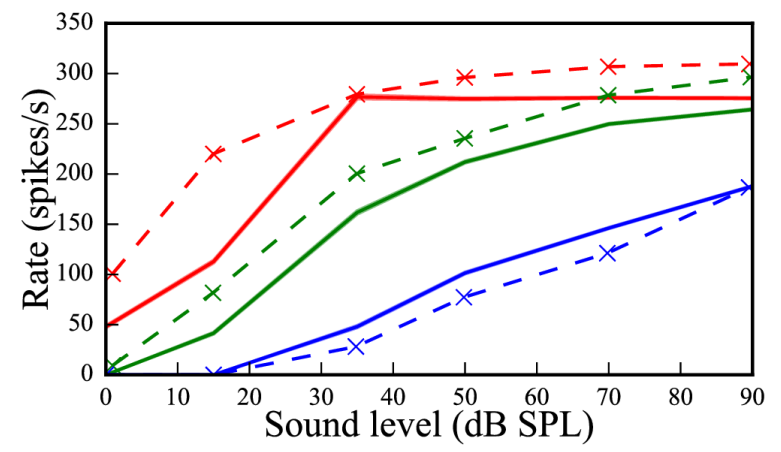

$\mathrm{B}$

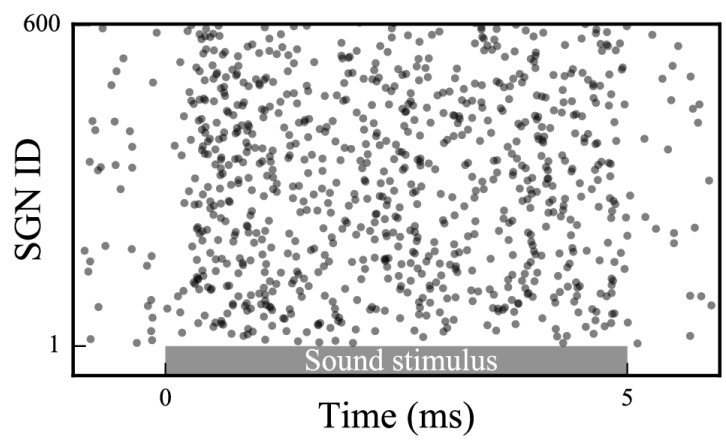

$\mathrm{D}$

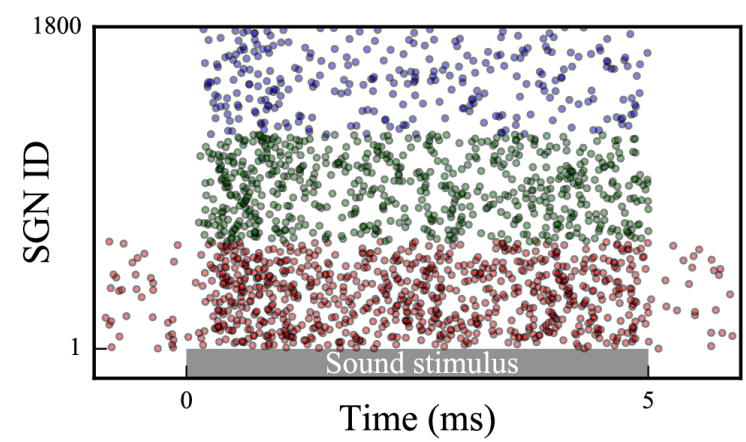

$\mathrm{F}$

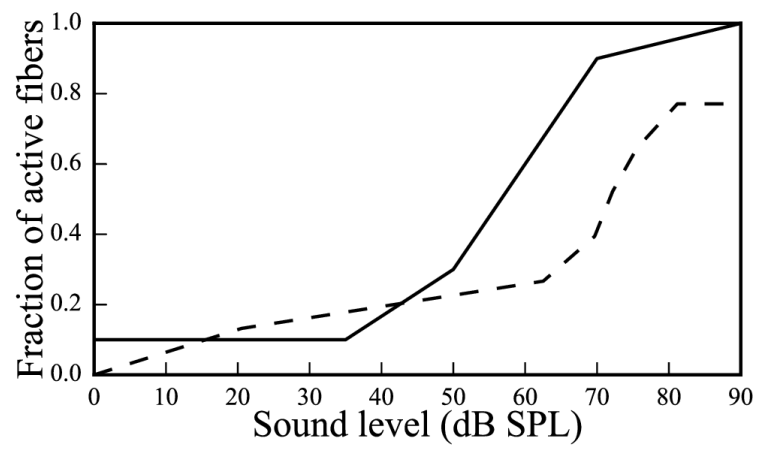

\begin{tabular}{|llll}
- & Low threshold - & Medium threshold - High threshold \\
\hline
\end{tabular}

169 Fig 2. Sound-evoked activity of low, medium and high threshold SGN fibers results from increased vesicle release probabilities from corresponding IHC-SGN synapses

171 (A) Sound stimuli is modeled as an increased vesicle release probability from IHCs where release

172 times are determined by a Poisson process ( $\mathrm{p}_{\text {spont: }}$ spontaneous release probability, $\mathrm{p}_{\text {peak: }}$ : maximum

173 release probability, phalf: release probability after adaptation, $t_{\text {start: }}$ sound start time, $t_{\text {stop}}$ : sound end

174 time, thalf: adaptation start time). For each release event, the corresponding SGN fiber is stimulated

175 with a brief external current pulse, resulting in spiking activity; cumulative activity of an SGN

176 fiber population is shown in (B), where gray dots represent spike times of each SGN fiber in a 177 population defined as different SGN fiber IDs. (C) Three groups of SGN fibers, low (LT), medium 
178 (MT) and high (HT) threshold, were simulated based on their spontaneous firing rates and saturation profiles in response to sound, by defining $p_{\text {peak }}$ and $p_{\text {spont }}$ for each fiber type at each

180 sound level. (D) Based on the release probabilities, different fiber types exhibit different 181 cumulative responses (red dots: low threshold, green dots: medium threshold, blue dots: high 182 threshold). Panels A-D are example simulations for simulated 50dB SPL. (E) The trend of spike 183 rates of each fiber type for various sound levels in our model (solid lines) are comparable to 184 experimental results (dashed lines) (Data taken from [19]). (F) For higher sound levels, due to the 185 recruitment of SGN fibers with CFs near that of the simulated sound frequency, the fraction of 186 activated SGN fibers increases for sound levels higher than 35dB SPL. At 90dB SPL, all 6000 187 fibers are activated (solid line: our model, dashed line: calculated based on spiral ganglion cell 188 densities for different CFs [21] and IHC tuning curves [22]).

191 IHC release that governed brief external stimuli to the corresponding nerve fiber to induce action

192 potential generation. The external stimuli mimicking synaptic release from IHCs were simulated

193 in the form of external current pulses with amplitude $0.024 \mathrm{nA}$ and duration $0.05 \mathrm{~ms}\left(\mathrm{I}_{\mathrm{app}}\right)$ applied

194 at the beginning of the unmyelinated segment, unless otherwise stated (S3A Fig). The amplitude

195 and duration of the stimuli were chosen to be close to the threshold stimulation needed to result in

196 action potential generation at the heminode of putative control $\left(\mathrm{L}_{\mathrm{u}}=10 \mu \mathrm{m}, \mathrm{L}_{\mathrm{h}}=1 \mu \mathrm{m}\right)$ fibers. The

197 time of the action potential at the center of the heminode was taken as output (Fig 2B).

198 In our model, to reproduce experimentally observed shape of the stimulus mediated release

$199[6]$, we used $5 \mathrm{~ms}$ long sound stimuli $\left(t_{\text {stop }}-t_{\text {start }}=5 \mathrm{~ms}\right)$ and set the time from stimulus

200 initiation to release probability reaching phalf, i.e. $\left(t_{\text {half }}-t_{\text {start }}\right)$, to $1.4 \mathrm{~ms}[20]$. We varied $p_{\text {spont }}$ and

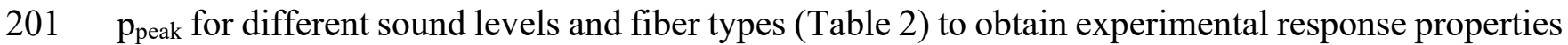

$202[19]$. 
Table 2: Spontaneous ( $\left.p_{\text {spont}}\right)$ and maximum ( $\left.p_{\text {peak }}\right)$ release probabilities for low- (LT), medium- (MT) and high-threshold SGN fibers (HT)

\begin{tabular}{|c|c|c|c|c|}
\hline & & $\mathbf{L T}$ & MT & HT \\
\hline \multicolumn{2}{|r|}{ pspont $_{\text {spo }}$} & 0.00025 & 0 & 0 \\
\hline \multirow{6}{*}{ 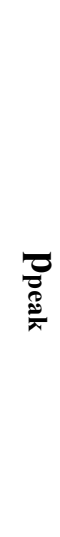 } & $0 \mathrm{~dB}$ SPL & 0 & 0 & 0 \\
\hline & $15 \mathrm{~dB}$ SPL & 0.0007 & 0.0004 & 0 \\
\hline & $35 \mathrm{~dB}$ SPL & 0.0027 & 0.0017 & 0.00045 \\
\hline & $50 \mathrm{~dB}$ SPL & 0.0027 & 0.0023 & 0.001 \\
\hline & $70 \mathrm{~dB}$ SPL & 0.0027 & 0.0028 & 0.0015 \\
\hline & $90 \mathrm{~dB}$ SPL & 0.0027 & 0.003 & 0.002 \\
\hline
\end{tabular}

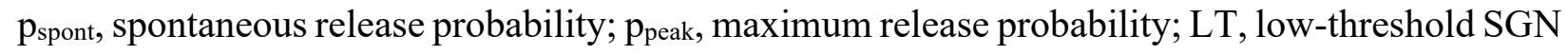
fiber; MT, medium-threshold SGN fiber; HT, high-threshold SGN fiber

222 thresholds for sound-evoked activity and saturation profiles, namely low threshold (LT), medium 223 threshold (MT) and high threshold (HT) fibers. Based on the measurements reported in [19], we 224 modeled the properties of these three fiber groups as follows (Figs 2C-E): LT fibers have high 225 spontaneous rates (18-100 spikes/s), low dynamic ranges, and reach their maximum discharge rate within approximately $30 \mathrm{~dB}$ sound pressure level (SPL). MT fibers have lower spontaneous firing

227 (between 0.5 and 18 spikes/s), higher dynamic ranges, and show slower increase and saturation of 228 spike rates with increasing SPL compared to LT fibers. HT fibers have very low spontaneous firing 229 rates $(<0.5$ spikes/s), and response thresholds higher than $\sim 20 \mathrm{~dB}$ SPL. For higher SPL, their spike 230 rate increases linearly with sound intensity, therefore their dynamic range is the highest [19]. 


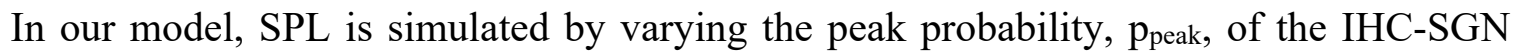
synaptic release probability function. The fiber type response properties are obtained by defining

233 spontaneous release probability, $\mathrm{p}_{\text {spont, }}$ and scaling $\mathrm{p}_{\text {peak }}$ for each sound level. Figs $2 \mathrm{C}$ and $2 \mathrm{D}$ show

234 IHC-SGN synaptic release probability functions and spike firing, respectively, for 70dB SPL for

235 each fiber type (see Table 2 for all sound levels). For simplicity, we assumed MT and HT fibers

236 do not fire spontaneously.

237 The intensity of sound stimuli affects the number of recruited type I SGNs as well. At lower 238 sound levels, only the fibers with the characteristic frequencies (CFs) close to the stimulus fire. At 239 higher SPL, the spatial profile of excitation spreads, and fibers with a broader range of CFs also 240 respond to the stimulation. To introduce the recruitment of more fibers with increasing SPL, we 241 considered the IHC tuning curves [22] and the density of SGNs based on their CF [21], and used 242600 fibers for sound intensities of 35dB SPL and lower, 1800 fibers for $50 \mathrm{~dB}$ SPL, 5400 fibers 243 for $70 \mathrm{~dB}$ SPL and 6000 for $90 \mathrm{~dB}$ SPL, with equal numbers of LT, MT and HT fibers (Fig 2F).

245 Analyzing spike trains obtained from simulations

246 In response to simulated sound stimulus, each model SGN fiber fires a sequence of spikes (Fig

247 2D). We used three methods to analyze SGN fiber spike trains:

248 Measurement of time intervals between non-identical spike trains of SGN fiber populations.

249 This metric, modified from a shuffled autocorrelogram measure in [23], was used to quantify 250 temporal properties of SGN fiber spiking within a population based on the time intervals of the 251 spikes between each non-identical pair of spike trains within the population. From all possible 252 non-identical pairs of spike trains within a population, forward time intervals were measured 253 between each spike $i$ of the first spike train and spikes of the second spike train falling between 
254 the $i$-th and $(i+1)$-st spikes (Fig 3A). All time intervals from all pairs were tallied in a histogram

255 and the histogram was reflected over $y$-axis, since each forward time interval of a pair $(\mathrm{x}, \mathrm{y})$ is a

256 backward time interval of the pair $(y, x)$.

A

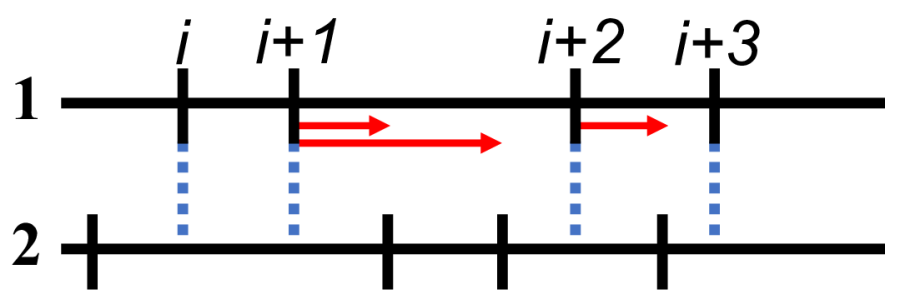

$\mathrm{B}$

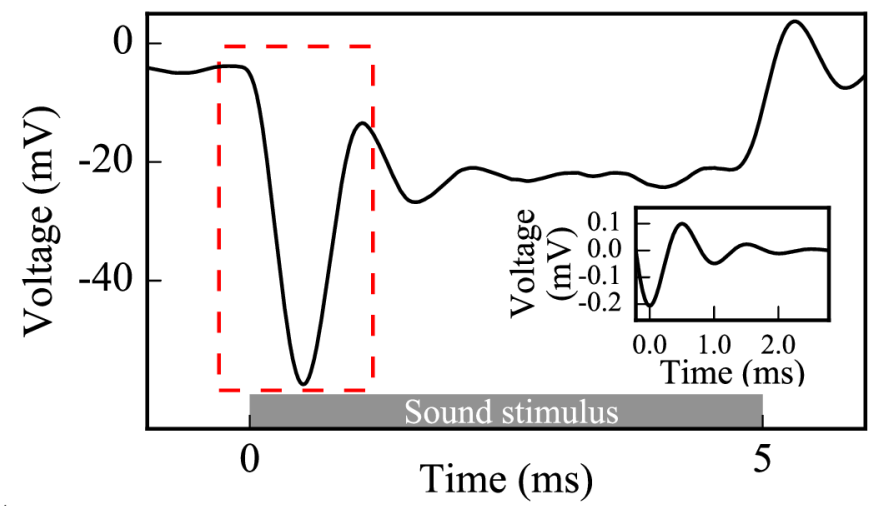

C

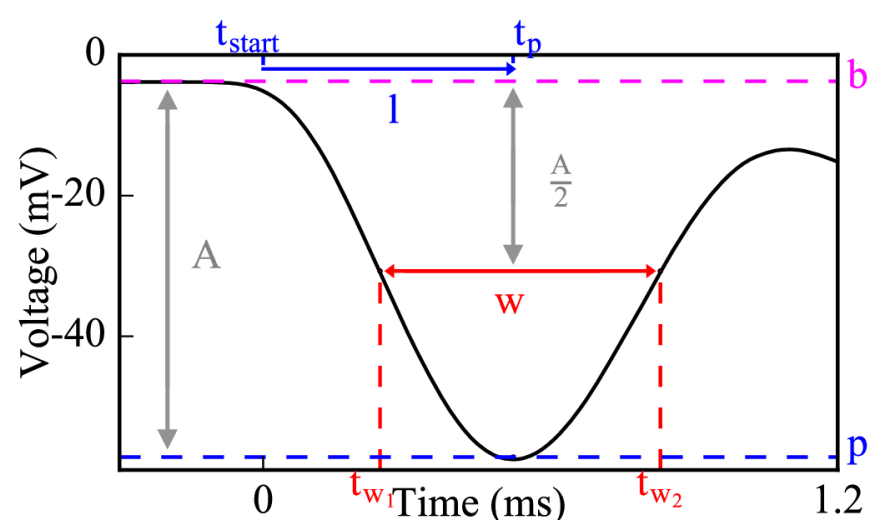

258 Fig 3. Methods used to evaluate cumulative activity of SGN fiber populations: pairwise spike time differences (A) and simulated CAP $(B, C)$.

260 (A) For each non-identical pair of spike trains from an SGN fiber population, forward time intervals are measured between each spike $i$ of the spike train 1 and all spikes of the spike train 2 falling between $i$ and $i+1$. Standard deviations of the distributions of these time intervals are calculated to evaluate synchronous spike timing in the SGN fiber population. (B) Each spike in 
264 Fig 2D is convolved with the unitary response of CAP [the inset of (B)] and convolutions from 265 each spike are summed up to obtain a simulated CAP of the SGN fiber population. (C) Amplitude, 266 latency and width are measured from the first peak of the simulated CAP [dashed rectangle in (B) 267 is zoomed in for $(\mathrm{C})$ ] (b: baseline, $\mathrm{p}$ : peak, A: amplitude of the peak, $\mathrm{t}_{\mathrm{p}}$ : peak time, l: latency, w: 268 width, $t_{\mathrm{w} 1}$ : half amplitude time before $t_{\mathrm{p}}, \mathrm{t}_{\mathrm{w} 2}$ : half amplitude time after $\mathrm{t}_{\mathrm{p}}$ ).

Convolution into the unitary response of compound action potential (CAP). To yield a

271 cumulative response of the activity of the population of SGN fibers and to be able to compare

272 model results with in vivo ABR P1 results, we convolved each spike with the unitary response and 273 summed them up to generate a population CAP (Fig 3B). In this study, we considered this 274 computed CAP as equivalent to ABR P1. The unitary response U(t) was described as in [24]:

$$
U(t)=\left\{\begin{array}{l}
A \times e^{-k(t-0.288)} \times \sin (2 \pi f(t-0.288)) \quad \text { for }-0.215 \leq t \leq 2.785 \\
0
\end{array}\right.
$$

otherwise

276 where $A=0.14 \mu V, k=1.44 m s^{-1}, f=0.994 m s^{-1}$ and $t$ is the time (Fig 3B inset).

(w) of the initial CAP peak more accurately, which were computed as:

$$
\begin{gathered}
a=|p-b| \\
l=t_{p}-t_{\text {start }} \\
w=t_{w 2}-t_{w 1}
\end{gathered}
$$

where $\mathrm{p}$ is the peak voltage, $\mathrm{b}$ is the baseline voltage, $\mathrm{t}_{\mathrm{p}}$ is the time when the voltage equals $\mathrm{p}$, and

$283 t_{\mathrm{w} 1}$ and $t_{\mathrm{w} 2}$ are the times when the voltage equals $-\left(|b|+\frac{a}{2}\right)$ (the half-peak) before and after $t_{\mathrm{p}}$ 
Calculating spike probability and latency for each SGN fiber population. The probability that release events at IHC-SGN synapses resulted in spikes at the heminodes of an SGN fiber population was calculated by dividing the number of spikes at the heminode of each SGN fiber by the number of release events and averaging over all fibers within a population. Spike latency of an SGN fiber population was calculated by the time difference between a spike and a release preceding that spike averaged over all spikes of that population.

\section{Results}

Using the model of the type I SGN fiber population, we investigated the effects of segment $\mathrm{L}_{\mathrm{u}}($ Fig $1 \mathrm{C}$, from a putative control value of $10 \mu \mathrm{m})$ and the first heminode length $\mathrm{L}_{\mathrm{h}}($ from a control value of $1 \mu \mathrm{m}$ ) for all (i.e. LT, MT and HT) fibers. Next, we simulated synaptopathy by removing IHC-SGN synapses (Fig 1B) considering the cases where only synapses on HT fibers are affected or synapses on all fiber types are affected. Lastly, we investigated the combined effects

301 of myelinopathy and synaptopathy.

\section{Effects of myelinopathy on SGN population activation patterns}

Mouse studies have shown that transient demyelination and the subsequent remyelination

306 IHC-SGN synapse and at variable positions, in contrast to healthy SGN fibers where heminodes

307 on all fibers are aligned [6]. To identify the effect of this heterogeneity of heminode locations on

308 SGN spike timing, we first considered a population of only LT fibers with different ranges of $\mathrm{L}_{u}$ 
309 values (Fig 4). Here, we denote $0 \%$ increase as the putative control fiber length $\left(\mathrm{L}_{\mathrm{u}}=10 \mu \mathrm{m}\right)$, while

$310100 \%$ increase means $\mathrm{L}_{\mathfrak{u}}$ was varied between 10 and $20 \mu \mathrm{m}$ across the population. We assessed the

311 level of synchronization of spikes across the SGN fiber population by stimulating all fibers with

312 the same $90 \mathrm{~dB}$ simulated SPL with the identical IHC release pattern. As heterogeneity of $\mathrm{L}_{\mathrm{u}}$ values

313 was increased (Fig 4A), the population spike rate decreased reflecting spike generation failure on

314 fibers with large $\mathrm{L}_{\mathrm{u}}$. At the same time, variability in spike timing increased as illustrated in spike

315 raster plots (Figs 4B, D, F, H show a portion of the generated spike trains, insets show timing of

316 first spikes) and computed pairwise spike time intervals (Figs 4C, E, G, I, see Methods). These

317 disruptions in spike generation and timing resulted in increased standard deviation of the

318 distribution of pairwise spike time differences across the population (Fig 4A). These initial

319 observations suggest that myelinopathy not only disrupts spike timing of SGNs within a

320 population, but also leads to the loss of spikes. 


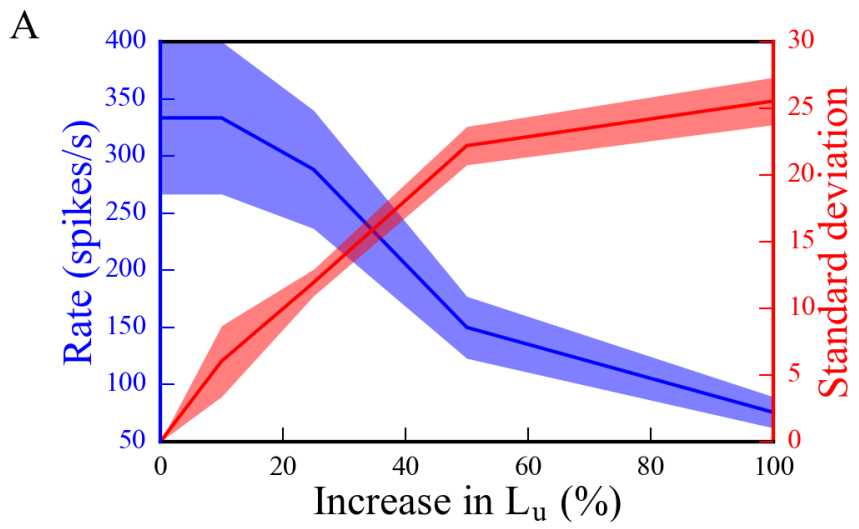

B

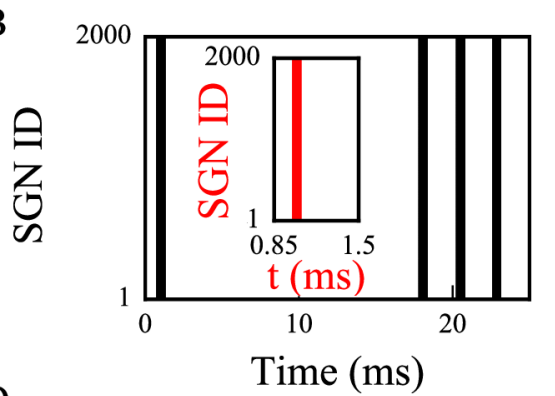

$\mathrm{D}$

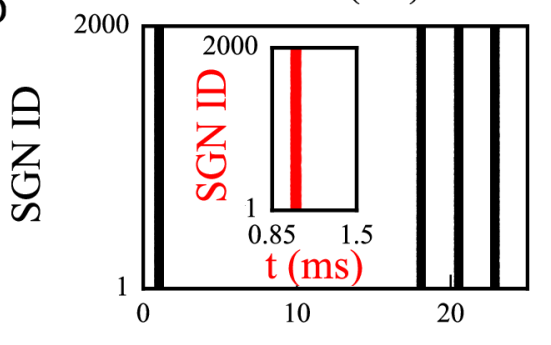

Time (ms)

$\mathrm{F}$

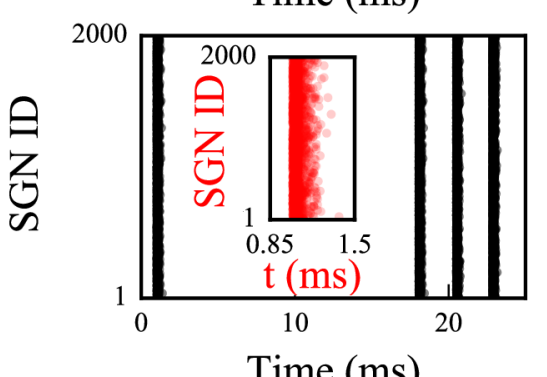

$\mathrm{H}$

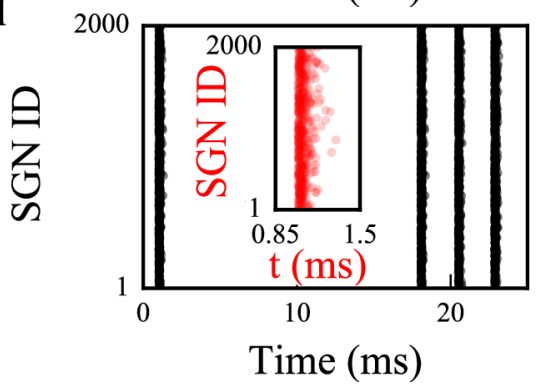

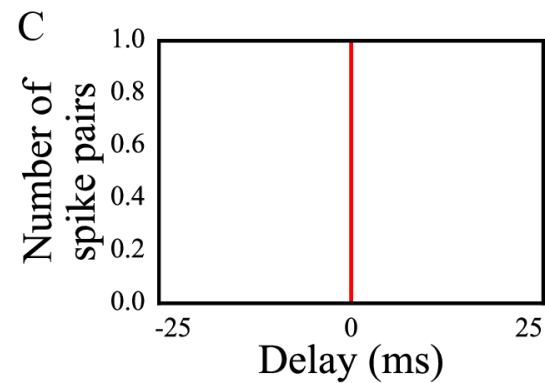

$\mathrm{E}$
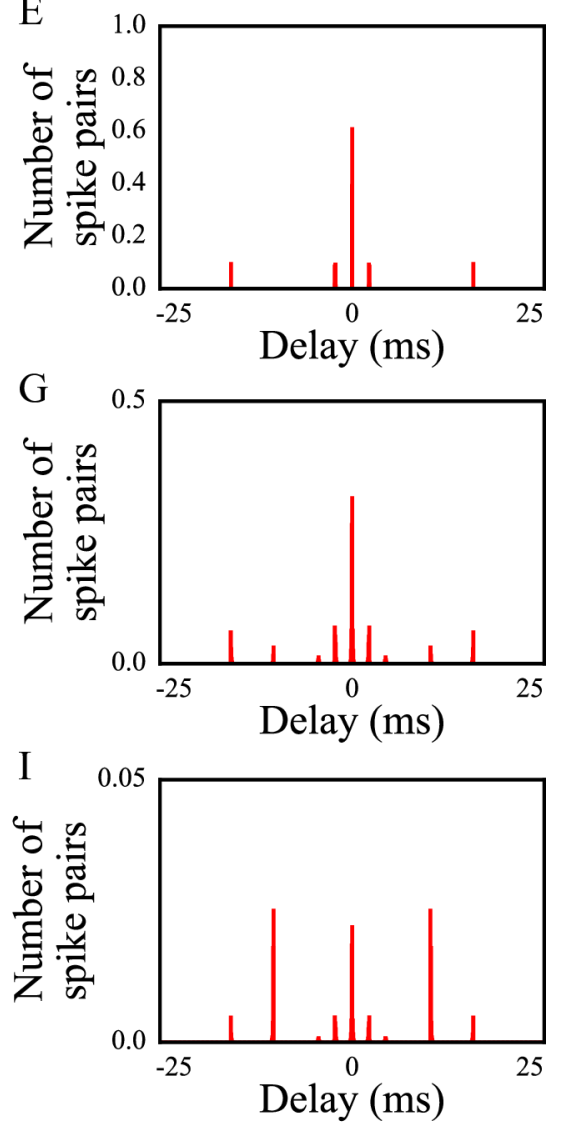

Fig 4. The synchronous activity of SGN fiber populations is disrupted and their response to 
SGN fiber populations with different heterogeneity levels of $\mathrm{L}_{\mathrm{u}}$ were stimulated using the identical simulated 90dB SPL stimulus. We assumed release events from all IHCs for the population occurred simultaneously. Raster plots of a portion of the generated spike trains [(B), (D), (F) and $(\mathrm{H})$, insets: timings of first spikes] and corresponding histograms of time intervals between nonidentical pairs of spike trains within a population $[(\mathrm{C}),(\mathrm{E}),(\mathrm{G})$ and $(\mathrm{I})]$ are shown for populations of SGN fibers with $\mathrm{L}_{\mathrm{u}}=10 \mu \mathrm{m}\left(0 \%\right.$ increase in $\left.\mathrm{L}_{\mathrm{u}}\right)[(\mathrm{B})$ and $(\mathrm{C})], 10 \mu \mathrm{m} \leq \mathrm{L}_{\mathrm{u}} \leq 11 \mu \mathrm{m}(10 \%$ increase in $\left.\mathrm{L}_{\mathrm{u}}\right)[(\mathrm{D})$ and $(\mathrm{E})], 10 \mu \mathrm{m} \leq \mathrm{L}_{\mathrm{u}} \leq 12.5 \mu \mathrm{m}\left(25 \%\right.$ increase in $\left.\mathrm{L}_{\mathrm{u}}\right)[(\mathrm{F})$ and $(\mathrm{G})]$ and $10 \mu \mathrm{m} \leq \mathrm{L}_{\mathrm{u}} \leq 20 \mu \mathrm{m}$ $\left(100 \%\right.$ increase in $\left.\mathrm{L}_{\mathrm{u}}\right)[(\mathrm{H})$ and $(\mathrm{I})]$. The ordinates of the histograms are normalized over the number of spike pairs with $0 \mathrm{~ms}$ delay for the population where all fibers have $\mathrm{L}_{\mathrm{u}}=10 \mu \mathrm{m}(\mathrm{C})$. Simulations were done 3 times. Firing rate and standard deviations of time intervals are averaged for all populations in (A), shaded area represents the standard error of the mean.

To investigate effects of this disruption of spike generation and timing in the full model,

to simulated myelinopathy. Responses of fiber populations with homogeneous initial unmyelinated segments $\left(\mathrm{L}_{\mathrm{u}}\right)$ or first heminode length $\left(\mathrm{L}_{h}\right)$ values were investigated to see the gradual effect of variable myelination patterns on cumulative activity of SGN fibers. Additionally, populations with heterogeneous, random $\mathrm{L}_{\mathrm{u}}$ or $\mathrm{L}_{\mathrm{h}}$ values were simulated to represent a population heterogeneity induced by myelinopathy. We note that when increasing first heminode length $\left(\mathrm{L}_{\mathrm{h}}\right)$

343 the number of expressed channels $\left(\mathrm{Na}^{+}\right.$and $\left.\mathrm{K}^{+}\right)$was kept constant consequently decreasing their

344 density. However, when increasing initial unmyelinated segment length $\left(\mathrm{L}_{\mathrm{u}}\right)$, the density of 345 expressed channels was kept constant consequently increasing their number. Results were not qualitatively different when these assumptions were reversed (see Discussion section). Model

347 results show that, in response to a simulated $70 \mathrm{~dB}$ SPL stimulus, CAPs computed from SGN fiber 348 populations with homogeneous myelination patterns had decreased peak amplitude and increased

349 latency to the peak when $\mathrm{L}_{\mathrm{u}}$ was longer than the putative normal length of $10 \mu \mathrm{m}(\mathrm{Fig} 5 \mathrm{~A})$ and $\mathrm{L}_{\mathrm{h}}$

350 was longer than the putative normal length of $1 \mu \mathrm{m}$ (Fig 6A). The amplitude decrease was highly

351 significant for $\mathrm{L}_{\mathrm{u}}>12 \mu \mathrm{m}$ and $\mathrm{L}_{\mathrm{h}}>3 \mu \mathrm{m}$ with $\sim 80 \%$ of a drop from normal without or with 
352 including recruitment of additional fibers with increasing SPL (Figs 5B, C and 6B, C, 353 respectively). This was due to the fact that at those values failure of spike generation occured

354 because of the increased lengths, $\mathrm{L}_{u}$ and $\mathrm{L}_{h}$. CAP peak latencies were significantly longer than 355 normal for all homogeneous populations, with $\mathrm{L}_{\mathrm{u}}>12 \mu \mathrm{m}$ and $\mathrm{L}_{\mathrm{h}}>3 \mu \mathrm{m}$ having $\sim 40 \%$ of an

356 increase. The changes in CAP widths were minimal for all cases, and only significant when $\mathrm{L}_{\mathrm{u}}=13$

$357 \mu \mathrm{m}$ or $\mathrm{L}_{\mathrm{u}}=14 \mu \mathrm{m}$ and $\mathrm{L}_{\mathrm{h}}=4 \mu \mathrm{m}$ or $\mathrm{L}_{\mathrm{h}}=5 \mu \mathrm{m}$ with additional fiber recruitment (Figs 5C and 6C).

358 For populations with heterogeneous myelination patterns, however, CAP peaks were significantly

$359(\sim 50 \%)$ lower, and latencies and widths were significantly higher than normal populations. 
A

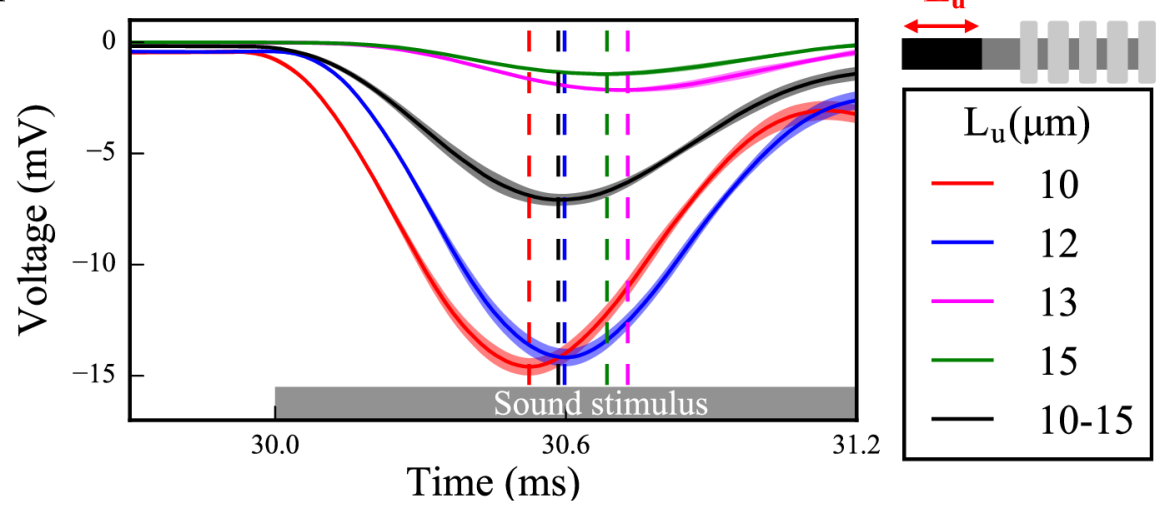

$\underline{\text { Without recruitment }}$

B



D

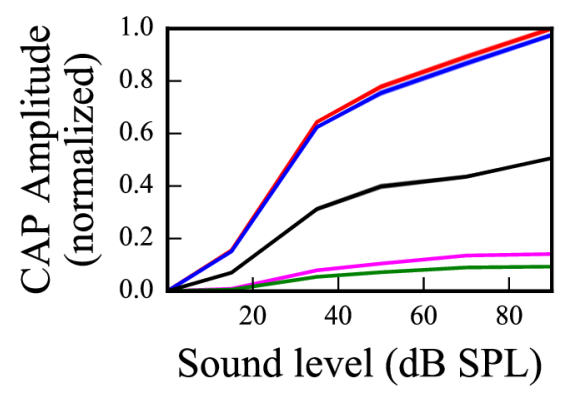

F

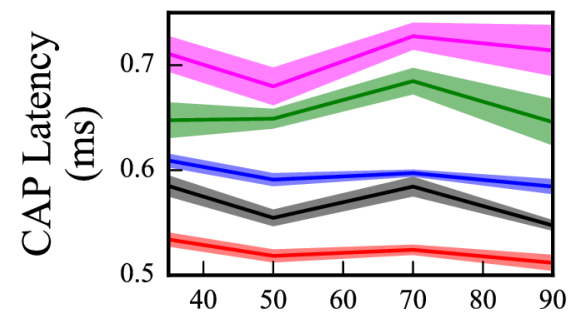

Sound level (dB SPL)
C

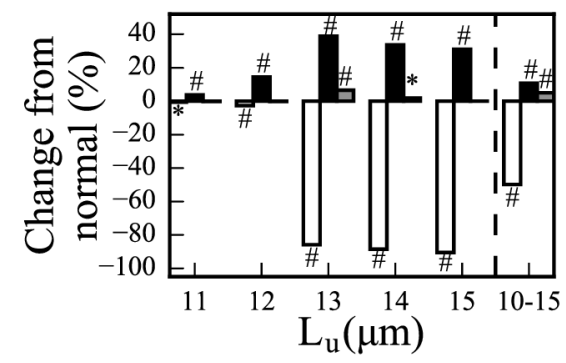

Latency

E

$\underline{\text { With recruitment }}$ C

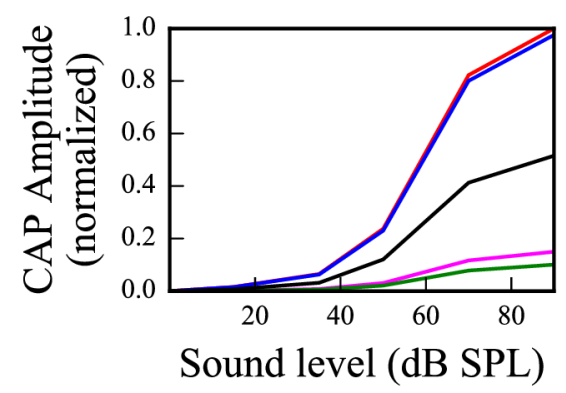

G

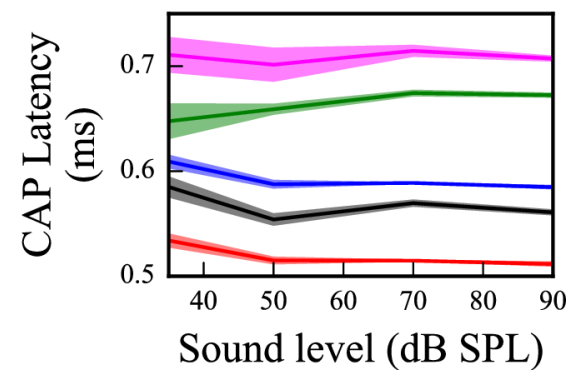

361 Fig 5. Longer $L_{u}$ significantly decreases and delays the peak of the sound-evoked CAPs of SGN fibers. 
363 (A) Sound-evoked CAPs of SGN fiber populations with varying $\mathrm{L}_{\mathfrak{u}}$ at $70 \mathrm{~dB}$ SPL without 364 recruitment of additional fibers, averaged over 50 simulations. Shaded regions correspond to the 365 standard error of the mean and dashed lines correspond to the peaks of each CAP, labeled with the 366 same colors as the CAPs. The decrease and delay of peak CAPs are more obvious for populations 367 with $\mathrm{L}_{u}>12 \mu \mathrm{m}$. Comparison of CAP measures of each population relative to normal $\mathrm{L}_{u}\left(\mathrm{~L}_{\mathrm{u}}=10\right.$ $368 \mu \mathrm{m}$ ) for cases without (B) and with (C) recruitment at $70 \mathrm{~dB}$ SPL. Latencies are significantly 369 higher for all populations and peaks are significantly lower for populations with $\mathrm{L}_{\mathrm{u}}>12 \mu \mathrm{m}$. The 370 increases in widths are only minimal, however significant for the heterogeneous population, where $37110 \mu \mathrm{m} \leq \mathrm{L}_{\mathrm{u}} \leq 15 \mu \mathrm{m}\left({ }^{*} \mathrm{p}<0.05,{ }^{*} \mathrm{p}<0.005\right.$, \#p $\left.<0.0005\right)$. Normalized CAP amplitudes without (D) 372 and with (E) recruitment for various sound levels show qualitatively similar behavior, but the case 373 with recruitment (E) exhibits a more exponential increase. The latencies of CAP peaks increase 374 with higher $\mathrm{L}_{\mathrm{u}}$ for all sound levels with no change along the sound levels, and similar values 375 without $(\mathrm{F})$ and with $(\mathrm{G})$ recruitment. 
A

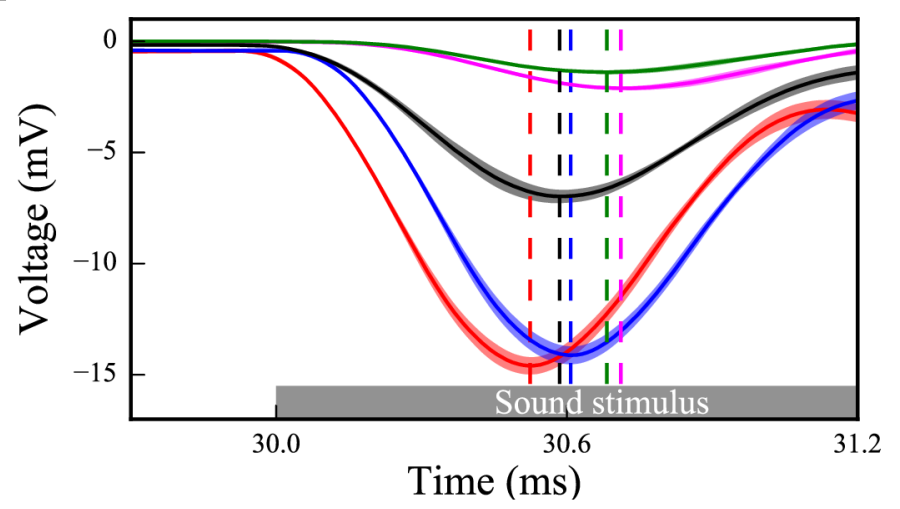

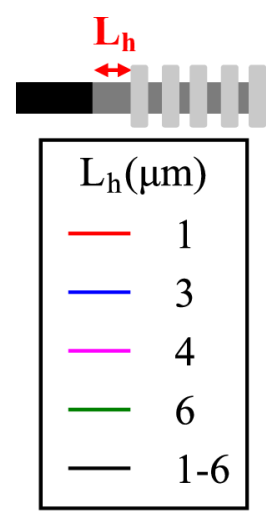

$\underline{\text { With recruitment }}$

C

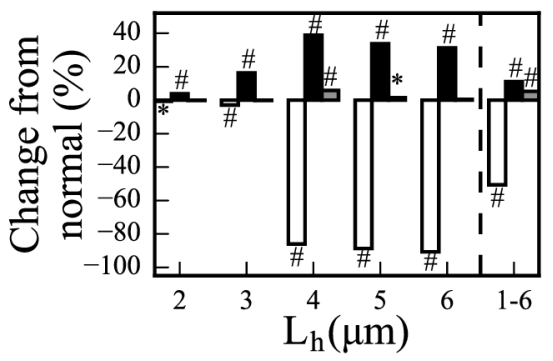

Latency Width

E

D

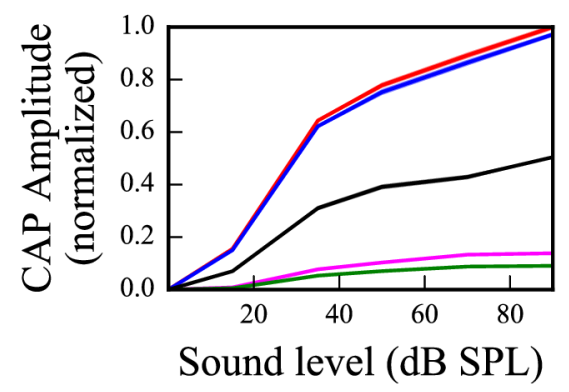

F

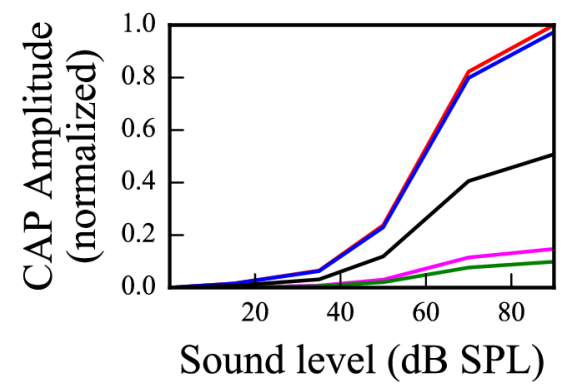

G

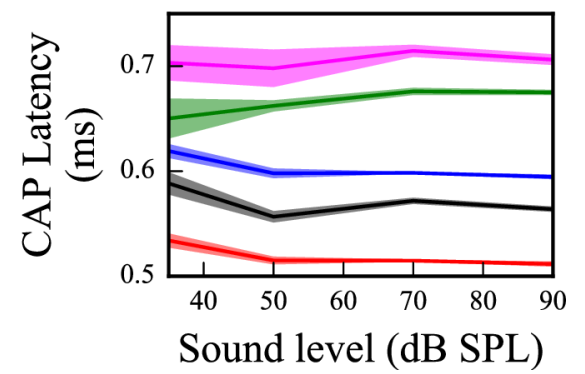



Sound level (dB SPL)
Sound level (dB SPL)

Sound level (dB SPL)

377 Fig 6. Longer $L_{h}$ significantly decreases and delays the peak of the sound-evoked CAPs of 
(A) Sound-evoked CAPs of SGN fiber populations of varying $L_{h}$ at 70dB SPL without recruitment of additional fibers, averaged over 50 simulations. Shaded regions correspond to the standard error of the mean and dashed lines correspond to the peaks of each CAP, labeled with the same colors as the CAPs. The decreased peak amplitude and increased latency of CAP peak are more obvious for populations with $\mathrm{L}_{\mathrm{h}}>3 \mu \mathrm{m}$. Comparison of CAP measures of each population relative to the normal $\mathrm{L}_{\mathrm{h}}\left(\mathrm{L}_{\mathrm{h}}=1 \mu \mathrm{m}\right)$ without $(\mathrm{B})$ and with $(\mathrm{C})$ recruitment at $70 \mathrm{~dB}$ SPL. CAP latencies are significantly higher for all populations and peak amplitudes are significantly lower for populations with $\mathrm{L}_{\mathrm{h}}>3 \mu \mathrm{m}$. The increases in widths are only minimal, however significant for the heterogeneous population, where $1 \mu \mathrm{m} \leq \mathrm{L}_{\mathrm{h}} \leq 6 \mu \mathrm{m}\left({ }^{*} \mathrm{p}<0.05,{ }^{*} \mathrm{p}<0.005, \# \mathrm{p}<0.0005\right)$. Normalized CAP amplitudes without (D) and with (E) recruitment for various sound levels show qualitatively similar behavior, but the case with recruitment (E) exhibits a more exponential increase. The latencies of CAP peaks increase with higher $\mathrm{L}_{\mathrm{h}}$ for all sound levels with no change along the sound

In addition, to assess the dependencies of CAP properties on sound intensities, we measured responses to simulated sound stimuli between 0-90 dB SPL with and without additional fiber recruitment. For $\mathrm{L}_{\mathrm{u}} \leq 12 \mu \mathrm{m}$ and $\mathrm{L}_{\mathrm{h}} \leq 3 \mu \mathrm{m}$, CAP peak amplitudes increased with sound intensity (Figs 5D and 6D, respectively) and when fiber recruitment was included (Figs 5E and $6 \mathrm{E}$, respectively) the profile of increase was more similar to experimental measurements (see Supplementary Fig 4 in [6]). However, for $L_{u}>12 \mu \mathrm{m}$ and $L_{h}>3 \mu \mathrm{m}, \mathrm{CAP}$ amplitudes remained small for all sound intensities, with and without recruitment, due to reduced spike generation. For populations with heterogeneous myelination patterns, CAP amplitudes were between the $\mathrm{L}_{\mathrm{u}}=12$

$401 \mu \mathrm{m}$ and $\mathrm{L}_{\mathrm{u}}=13 \mu \mathrm{m}$ cases, and the $\mathrm{L}_{h}=3 \mu \mathrm{m}$ and $\mathrm{L}_{h}=4 \mu \mathrm{m}$ cases for all sound levels, reflecting 402 reduced spike generation in some fibers of the population with higher $\mathrm{L}_{\mathrm{u}}$ and $\mathrm{L}_{\mathrm{h}}$ values. CAP 403 latencies were longer for higher values of $\mathrm{L}_{\mathrm{u}}$ and $\mathrm{L}_{\mathrm{h}}$ with (Figs $5 \mathrm{G}$ and $6 \mathrm{G}$ ) or without (Figs $5 \mathrm{~F}$ 404 and 6F) recruitment, but did not exhibit significant changes with varying sound level. In the heterogeneous populations, CAP latencies showed values between the $\mathrm{L}_{\mathrm{u}}=10 \mu \mathrm{m}$ and $\mathrm{L}_{\mathrm{u}}=12 \mu \mathrm{m}$ cases and the $\mathrm{L}_{h}=1 \mu \mathrm{m}$ and $\mathrm{L}_{h}=3 \mu \mathrm{m}$ cases. 


\section{Effects of synaptopathy on SGN population activation patterns}

There is strong evidence indicating that noise-induced synaptopathy, primarily at HT

410 fibers, is one of the mechanisms of hidden hearing loss [8]. To simulate it, we considered responses

411 of a population of control SGN fibers $\left(\mathrm{L}_{\mathrm{u}}=10 \mu \mathrm{m}, \mathrm{L}_{\mathrm{h}}=1 \mu \mathrm{m}\right)$ with $50 \%$ of HT IHC-SGN synapses

412 removed. To investigate the specific effect of loss of synapses on HT fibers, we compared

413 responses to the case where the same number of synapses ( $1 / 6^{\text {th }}$ of whole population) were

414 removed randomly from the whole population of three fiber types. The CAPs computed from

415 populations with and without synaptopathy (Fig 7A) in response to a $70 \mathrm{~dB}$ SPL suggest that HT-

416 targeted synaptopathy produces only a small effect on CAP peak amplitude while random

417 synaptopathy has a much broader and significant effect on the amplitude ( $\sim 80 \%$ vs $\sim 10 \%$ decrease

418 from normal at 70dB SPL) (Figs 7B and C). Moreover, there was no latency and width changes

419 for HT synaptopathy with or without fiber recruitment. However, random synaptopathy

420 significantly increased width and latency with recruitment at 70dB SPL, even though the increase

421 was minimal $(<1 \%)$. 
A

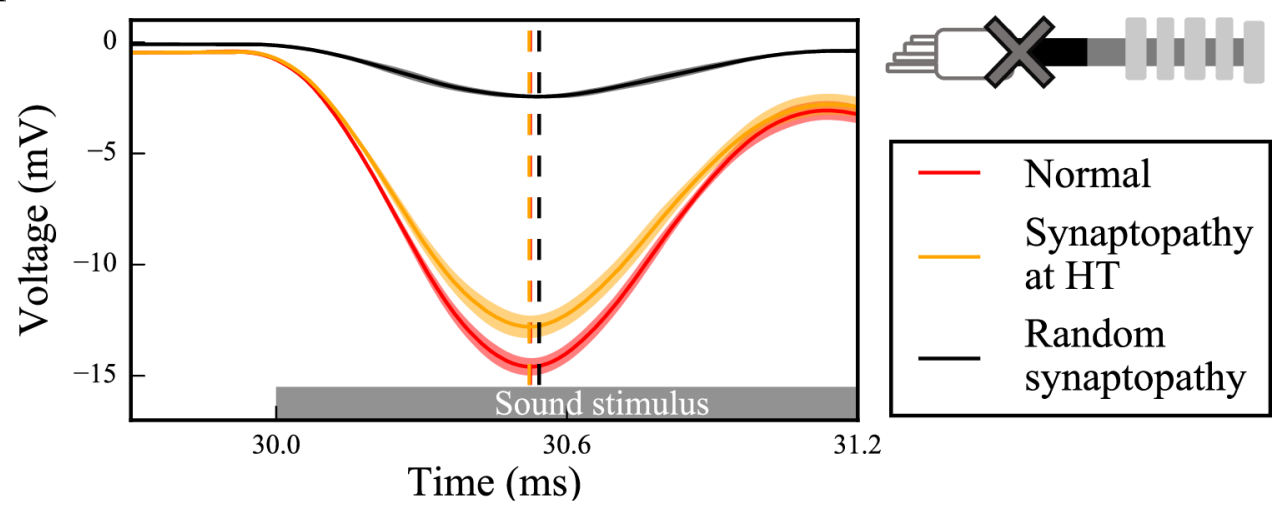

$\underline{\text { Without recruitment }}$

$\underline{\text { With recruitment }}$

B

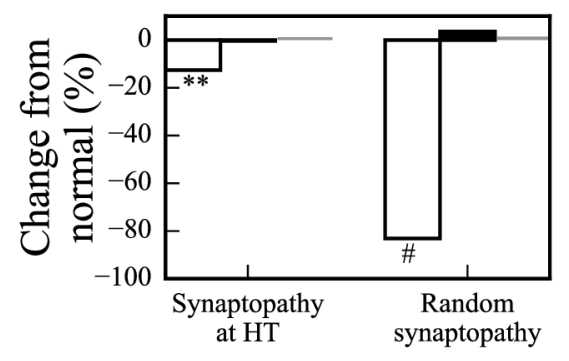

C



D

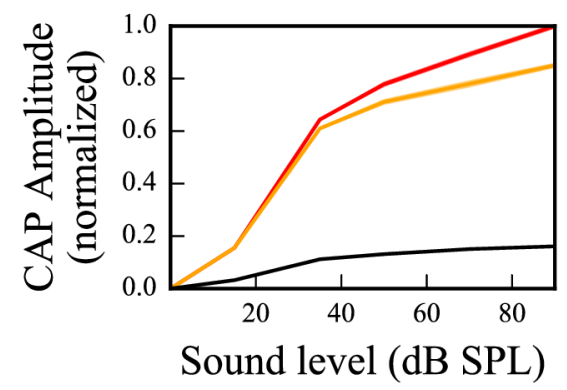

F

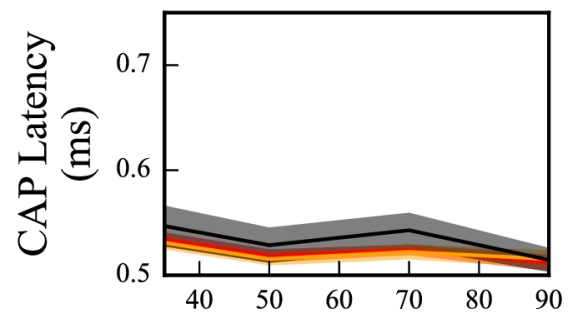

422
E

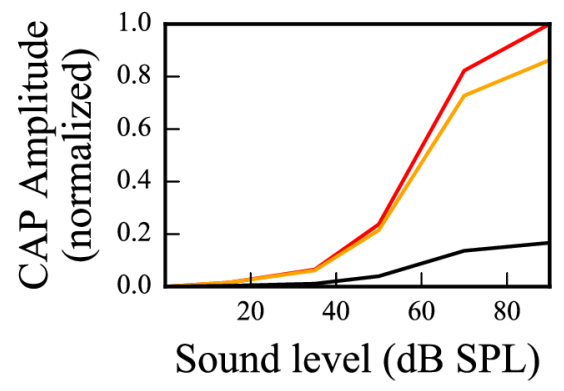

G

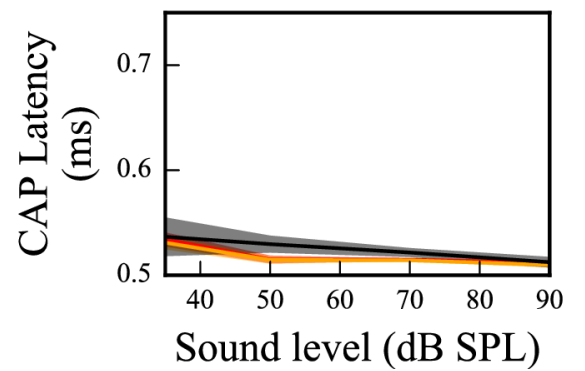

423 Fig 7. Synaptopathy at IHC-SGN synapses decreases the peak of the CAP significantly, 424 without changes to peak latency and width. 
(A) Sound-evoked CAPs of SGN fiber populations with different synaptopathy scenarios at 70dB SPL without recruitment of additional fibers, averaged over 50 simulations. Shaded regions correspond to the standard error of the mean and dashed lines correspond to the peaks of each CAP, labeled with the same colors as the CAPs. Synaptopathy has smaller effects on CAP peak amplitude and latency when it affects only HT fiber synapses compared to affecting all fiber types randomly. Comparison of CAP measures of synaptopathy cases relative to normal (no synaptopathy) without (B) and with (C) recruitment at $70 \mathrm{~dB}$ SPL $(* p<0.05, * * p<0.005$, $\# \mathrm{p}<0.0005)$. Normalized CAP amplitudes show qualitatively similar behavior without (D) and with (E) recruitment for various sound levels, but the case with recruitment exhibits a more exponential increase. The latencies of the CAP peaks do not exhibit any significant difference between without $(\mathrm{F})$ and with $(\mathrm{G})$ recruitment cases.

We simulated sound intensities between 0-90 dB SPL with and without recruitment to assess how CAP peak amplitude and latency depend on sound intensities in the synaptopathic cochlear model. For random synaptopathy CAP peaks remained small for all sound intensities while for HT synaptopathy, a decrease of CAP peaks was observed for higher sound intensities.

441 These results hold in cases with (Fig 7E) and without (Fig 7D) recruitment, but the profiles of CAP 442 peak amplitudes are more consistent with experimental observations when fiber recruitment was 443 included (see Supplementary Fig 4 in [6]). CAP latencies did not show any significant differences

444 for any sound level in any synaptopathy case with or without recruitment (Figs 7F and G).

446 Combined effects of myelinopathy and synaptopathy of hidden hearing loss

448 activity, we combined them in our model (Fig 8). When HT synaptopathy (Fig 8A and B) was 449 combined with myelinopathy affecting the length of the initial unmyelinated segment $\mathrm{L}_{\mathrm{u}}$, CAP 450 peak amplitude showed significant additive decrease but latency and width showed no change 451 beyond that produced by the myelin defects alone (compare Case 3 with Cases 1 and 2). When 452 both myelinopathy mechanisms were combined by varying $\mathrm{L}_{\mathrm{u}}$ and $\mathrm{L}_{\mathrm{h}}$ across the population, both 
453 CAP peak amplitude and latency showed significant additive changes (compare Case 4 with Case

454 2). CAP widths were significantly increased only by myelinopathy mechanisms, in response to

455 simulated 70dB SPL. In response to varied sound intensities between 0-90 dB SPL, the additive

456 effects of synaptopathy on CAP peak amplitude changes were prominent for higher SPL while

457 latencies showed little dependence on SPL (Figs 8C and D, respectively).

A

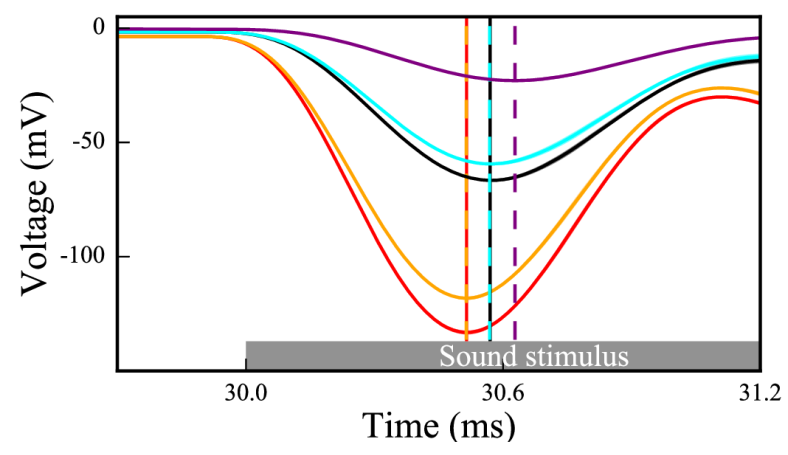

B

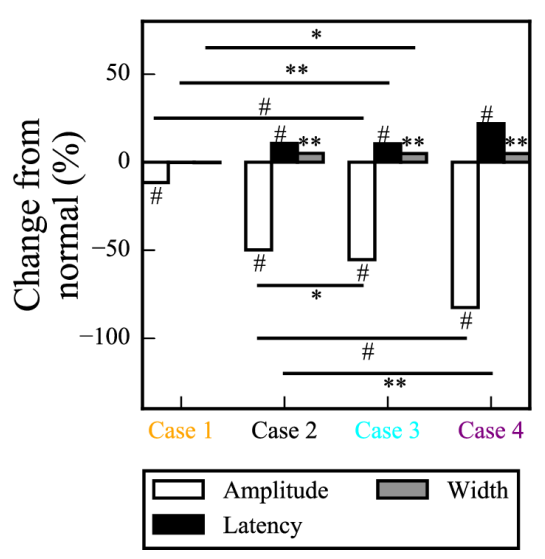

C

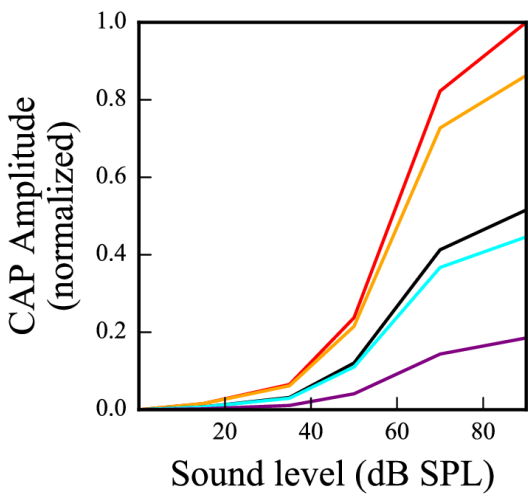

D



Fig 8. Different scenarios of hidden hearing loss have additive effects on SGN activity.

(A) Sound-evoked CAPs of SGN fiber populations with different myelinopathy and synaptopathy scenarios at 70dB SPL with recruitment of additional fibers, averaged over 50 simulations (dashed lines correspond to the peaks of each CAP, labeled with the same colors as the CAPs). Combined synaptopathy and myelinopathy (Case 3) shows additive effects on the decrease in CAP peak amplitude, but not on the increase in CAP peak latency (compare to Cases 1 and 2). Combined different myelinopathies show additive effects on both CAP peak amplitude and latency (compare Cases 2 and 4). (B) Comparison of average CAP measures for different myelinopathy and synaptopathy cases relative to normal, and between cases with recruitment at $70 \mathrm{~dB} \mathrm{SPL}(* \mathrm{p}<0.05$, 
myelinopathy and synaptopathy cases with recruitment for various sound levels, averaged over 50 simulations. Shaded areas correspond to the standard error of the mean.

473 effects for combined synaptopathy and myelinopathy. Also, there were significant increases in

474 CAP peak latencies and CAP widths only for myelinopathy-based mechanisms, with latencies

475 showing additive effects in combined myelinopathies, while synaptopathies do not affect this CAP

476 features.

\section{Discussion}

We built a reduced biophysical model simulating sound-evoked activity of type I SGN populations to analyze two hypotheses of the cause of HHL, synaptopathy and myelinopathy.

481 Model SGN spike times were convolved with the unitary response of the CAP, a near-field response of SGNs, to convert spike times into cumulative activity for comparison with experimental results. The model shows that synaptopathy reduces the amplitude of the cumulative

484 CAP response without affecting its latency due to a reduction in the number of nerve fibers responding without disruption of spike timing. In contrast, myelinopathy, when modeled as

486 disorganization of either the initial unmyelinated nerve segment length or the heminodal spacing, 487 causes disruption of spike timing in addition to loss of firing response, affecting both the peak 488 amplitude and latency of the cumulative CAP. Similar results are obtained when additional fibers, 489 associated with neighboring characteristic frequencies are recruited in response to high SPL 490 stimuli.

$491 \quad$ Previously, it has been shown that noise exposure and aging cause HHL due to synapse 492 loss at SGN-IHC synapses, which results in a decrease of ABR P1 without increases in latency or 
493 thresholds [4]. Moreover, it has been hypothesized that synapse loss occurs preferentially at HT

494 SGN-IHC synapses [8]. Consistent with experimental results, our simulations for both HT 495 synaptopathy and random synaptopathy show that CAP latencies are unchanged for either 496 scenario, but the amplitude of the CAP peak is significantly decreased. However, the decrease in 497 CAP amplitude was much larger for random synaptopathy and no significant activity was observed 498 for lower SPL stimuli, in contrast to HT synaptopathy case, where differences in SGN fiber activity 499 appeared only with higher SPL stimuli. These results suggest that synaptopathy at HT synapses is 500 a more likely scenario for HHL than random loss of synapses, since experimental results show that 501 thresholds remain unchanged (Fig 7) [8].

As shown by Wan and Corfas (2017), myelinopathy affects the distance from the IHC-

503 SGN synapse to the heminode and introduces heterogeneity in heminode locations across a SGN

504 fiber population, which is likely to result in their desynchronized activity [6]. Here, we provided

505 evidence that increasing heterogeneity of heminode locations decreases the synchronization of 506 spike timing of SGN fiber populations. Moreover, spike rates of more heterogeneous SGN fiber 507 populations dropped, suggesting a loss of spike generation in SGN fibers with heminodes further 508 from IHCs (Fig 4). Our simulations of cumulative CAP signals show that myelinopathy increases 509 the latency and the width of the peak of CAP, similar to experimental observations of ABR P1, 510 providing support for the disruption of spike timing in SGN activity (Figs 5 and 6). In addition, 511 the amplitude of the simulated CAP decreased with myelinopathy, reflecting the reduction of SGN 512 spike activity.

514 model. Decreases in CAP peak amplitude were additive for combined synaptopathy and 515 myelinopathy, but synaptopathy did not contribute to changes in CAP latency even in the 
516 combined scenario. Combining myelinopathy mechanisms led to additive increases in both peak

517 CAP amplitude and latency (Fig 8). These results match with the experimental results qualitatively,

518 further supporting the accuracy of our model.

519 In the myelinopathy simulations, we varied the length of the initial unmyelinated segment

$520 \mathrm{~L}_{\mathrm{u}}$ keeping a constant channel density (Fig 5) and varied the length of the heminode $\mathrm{L}_{\mathrm{h}}$ keeping

521 constant channel numbers (Fig 6). Results show similar effects on SGN fiber activity, i.e. the

522 populations with the same combined lengths $\mathrm{L}_{u}+\mathrm{L}_{h}$ exhibit the same behavior. As evidence on how

523 channels might be affected by the disruption of myelination patterns is lacking, we also simulated

524 cases where $\mathrm{L}_{\mathrm{u}}$ increases with constant channel number (S1 Fig) and $\mathrm{L}_{\mathrm{h}}$ increases with constant

525 channel density (S2 Fig). Results show that spreading the same number of channels over an

526 increased $\mathrm{L}_{\mathrm{u}}(\mathrm{S} 1 \mathrm{Fig})$, rather than increasing the number by keeping the channel density constant

527 (Fig 5), decreases the $\mathrm{L}_{\mathrm{u}}$ value at which the abrupt decrease in CAP peak occurs due to loss of

528 spike generation. With constant channel number, CAP peaks for homogeneous populations with

$529 \mathrm{~L}_{\mathrm{u}}>11 \mu \mathrm{m}$ decreased $\sim 90 \%$ from normal $\left(\mathrm{L}_{\mathrm{u}}=10 \mu \mathrm{m}\right)(\mathrm{S} 1 \mathrm{~B}$ Fig). However, the same drop occurred

530 when $\mathrm{L}_{\mathrm{u}}>12 \mu \mathrm{m}$ for the constant channel density case. In contrast, varying $\mathrm{L}_{\mathrm{h}}$ while keeping the

531 heminode channel density constant, i.e., increasing the number of channels for larger $\mathrm{L}_{\mathrm{h}}$, increased

532 the $\mathrm{L}_{\mathrm{h}}$ value associated with the loss of spike generation up to $6 \mu \mathrm{m}$, compared to $3 \mu \mathrm{m}$ when

533 channel number was kept constant (Fig 6). To conclude, any of these scenarios results in

534 qualitatively similar SGN fiber activity patterns, only affecting the $\mathrm{L}_{\mathrm{u}}$ and $\mathrm{L}_{\mathrm{h}}$ lengths at which loss

535 of spike generation leads to an abrupt drop in the CAP peak.

To better understand the effects of myelinopathy on SGN spike generation, we additionally

537 analyzed the population outcome of vesicle release events to the SGN fibers. As described in the

538 Methods section, SGN response to vesicle release was simulated by applying a brief external 
current pulse to the peripheral end of the SGN fibers. We thus calculated the probability that release events result in corresponding spikes for various amplitudes $\mathrm{I}_{\text {app }}$ of the external current

541 pulse for increasing values of $\mathrm{L}_{\mathrm{u}}\left(\mathrm{S} 3 \mathrm{~A}\right.$ Fig). For simulated 70dB SPL stimuli, higher $\mathrm{I}_{\mathrm{app}}$ amplitudes

542 increased spike probability for larger $L_{u}$ values, leading to increases in the $L_{u}$ values at which spike

543 generation was affected. If $\mathrm{L}_{\mathrm{u}}$ exceeded a critical value, the probability of spike generation

544 decreased significantly. These results show that this $\mathrm{L}_{\mathrm{u}}$ critical value required for spike generation depends on IHC-SGN synaptic efficacy.

To analyze the effect of sound level on SGN fiber spike probability, we ran simulations for

547 all sound levels keeping $\mathrm{I}_{\mathrm{app}}$ fixed at the default value $\left(\mathrm{I}_{\mathrm{app}}=0.024 \mathrm{nA}\right.$, solid black rectangle in $\mathrm{S} 3 \mathrm{~A}$

548 Fig). As described in the Methods section, increasing sound level was simulated by increasing the

549 probability of a vesicle release event, thus leading to higher rate of release from IHCs, i.e. higher

550 frequencies of external current pulse applications to SGN fibers. For this $\mathrm{I}_{\mathrm{app}}$ value, spike 551 generation was affected for $\mathrm{L}_{\mathrm{u}}>12 \mu \mathrm{m}$ as evident in the results shown in Fig 5. For SGN fibers 552 with $\mathrm{L}_{\mathrm{u}} \leq 12.3 \mu \mathrm{m}$, spike probabilities were higher than $70 \%$ for all sound levels (S3B Fig).

553 However, spike probabilities decreased gradually with higher sound levels due to the inability of 554 the fibers to respond to high frequency stimulation. This means, despite more frequent release 555 events from IHC-SGN synapses with higher sound levels, SGN fibers cannot fire with a higher 556 frequency due to the saturation of their spike rate, resulting in decreased spike probabilities. For 557 SGN fibers with $\mathrm{L}_{\mathrm{u}}>12.3 \mu \mathrm{m}$, spike probability was very low reflecting loss of spike generation 558 but it increased slightly with increasing sound level, as high frequency stimulation facilitated spike 559 generation due to temporal summation. Results for heterogeneous $\mathrm{L}_{\mathrm{u}}$ values between 10 and 15 $560 \mu \mathrm{m}$ showed intermediate spike probabilities ( 40\%) as compared to homogeneous $\mathrm{L}_{\mathrm{u}}$ values of 10 $561 \mu \mathrm{m}$, for all sound levels. 
Lastly, to analyze effects of myelinopathy on SGN spike latency, we averaged the time differences between each spike and the preceding release event causing the spike for populations

564 of SGN fibers with varied homogeneous $\mathrm{L}_{u}$ values and varied sound levels (S3C Fig). The

565 populations with $\mathrm{L}_{\mathrm{u}}>12 \mu \mathrm{m}$ were not included since spikes were not reliably generated and for the

566 heterogeneous population, the fibers with $\mathrm{L}_{\mathrm{u}}>12 \mu \mathrm{m}$ were ignored. The homogeneous populations

567 showed increased latencies with increasing $\mathrm{L}_{\mathrm{u}}$ and the heterogeneous population's latencies were

568 between those for $\mathrm{L}_{\mathrm{u}}=11 \mu \mathrm{m}$ and $\mathrm{L}_{\mathrm{u}}=12 \mu \mathrm{m}$. Latencies showed little dependence on sound levels.

569 However, standard deviations of spike latencies increased with sound level, presumably reflecting

570 higher variability in spike response to higher frequency stimulation (S3D Fig). Additionally, the

571 population with heterogeneous $\mathrm{L}_{\mathrm{u}}$ values showed higher standard deviations for all sound levels

572 than the homogeneous populations with $\mathrm{L}_{\mathrm{u}} \leq 12 \mu \mathrm{m}$. This increase in spike timing variability is

573 responsible for increases in the width of the cumulative CAP for the heterogeneous population

574 shown in Fig 5.

In conclusion, our model results show that HHL deficits due to myelinopathy could be

576 caused by not only loss of SGN spike activity, as in synaptopathy, but also disruption of spike

577 timing and synchronization across a population of SGN fibers. Illumination of the underlying

578 differences in these mechanisms for HHL based on the model may be useful for the development

579 and testing of treatments for HHL. Moreover, the model framework may be extended to investigate 580 mechanisms behind other peripheral auditory system disorders. 


\section{References}

583 1. Kohrman DC, Wan G, Cassinotti L, Corfas G. Hidden Hearing Loss: A Disorder with Multiple Etiologies and Mechanisms. Cold Spring Harb Perspect Med. 2019. Epub 2019/01/07. doi: 10.1101/cshperspect.a035493. PubMed PMID: 30617057.

2. Spankovich C, Gonzalez VB, Su D, Bishop CE. Self reported hearing difficulty, tinnitus, and normal audiometric thresholds, the National Health and Nutrition Examination Survey 19992002. Hear Res. 2017. Epub 2017/12/07. doi: 10.1016/j.heares.2017.12.001. PubMed PMID: 29254853.

3. Tremblay KL, Pinto A, Fischer ME, Klein BE, Klein R, Levy S, et al. Self-Reported Hearing Ear Hear. 2015;36(6):e290-9. doi: 10.1097/AUD.0000000000000195. PubMed PMID: 26164105; PubMed Central PMCID: PMCPMC4824300.

4. Kujawa SG, Liberman MC. Adding insult to injury: cochlear nerve degeneration after "temporary" noise-induced hearing loss. J Neurosci. 2009;29(45):14077-85. doi: 10.1523/JNEUROSCI.2845-09.2009. PubMed PMID: 19906956; PubMed Central PMCID: PMCPMC2812055.

5. Sergeyenko Y, Lall K, Liberman MC, Kujawa SG. Age-related cochlear synaptopathy: an early-onset contributor to auditory functional decline. J Neurosci. 2013;33(34):13686-94. doi: 10.1523/JNEUROSCI.1783-13.2013. PubMed PMID: 23966690; PubMed Central PMCID: PMCPMC3755715.

6. Wan G, Corfas G. Transient auditory nerve demyelination as a new mechanism for hidden hearing loss. Nat Commun. 2017;8:14487. Epub 2017/02/17. doi: 10.1038/ncomms14487. PubMed PMID: 28211470; PubMed Central PMCID: PMCPMC5321746. 
605 7. Rajan R, Cainer KE. Ageing without hearing loss or cognitive impairment causes a decrease in speech intelligibility only in informational maskers. Neuroscience. 2008;154(2):784-95. Epub 2008/04/04. doi: 10.1016/j.neuroscience.2008.03.067. PubMed PMID: 18485606.

608 8. Furman AC, Kujawa SG, Liberman MC. Noise-induced cochlear neuropathy is selective for fibers with low spontaneous rates. J Neurophysiol. 2013;110(3):577-86. Epub 2013/04/17.

9. Long P, Wan G, Roberts MT, Corfas G. Myelin development, plasticity, and pathology in the auditory system. Dev Neurobiol. 2018;78(2):80-92. Epub 2017/09/26. doi: 10.1002/dneu.22538. PubMed PMID: 28925106; PubMed Central PMCID: PMCPMC5773349.

10. Takazawa T, Ikeda K, Murata K, Kawase Y, Hirayama T, Ohtsu M, et al. Sudden deafness and facial diplegia in Guillain-Barré Syndrome: radiological depiction of facial and acoustic nerve lesions. Intern Med. 2012;51(17):2433-7. Epub 2012/09/01. PubMed PMID:

11. Choi JE, Seok JM, Ahn J, Ji YS, Lee KM, Hong SH, et al. Hidden hearing loss in patients with Charcot-Marie-Tooth disease type 1A. Sci Rep. 2018;8(1):10335. Epub 2018/07/09. doi: 10.1038/s41598-018-28501-y. PubMed PMID: 29985472; PubMed Central PMCID: PMCPMC6037750.

624 12. Kiang NY, Rho JM, Northrop CC, Liberman MC, Ryugo DK. Hair-cell innervation by spiral ganglion cells in adult cats. Science. 1982;217(4555):175-7. PubMed PMID: 7089553.

626 13. Hines ML, Carnevale NT. NEURON: a tool for neuroscientists. Neuroscientist. 2001;7(2):123-35. doi: 10.1177/107385840100700207. PubMed PMID: 11496923. 
628 14. Mino H, Rubinstein JT, Miller CA, Abbas PJ. Effects of electrode-to-fiber distance on temporal neural response with electrical stimulation. IEEE Trans Biomed Eng. 2004;51(1):13-20. doi: 10.1109/TBME.2003.820383. PubMed PMID: 14723489.

631 15. Møller AR, Colletti V, Fiorino FG. Neural conduction velocity of the human auditory nerve: bipolar recordings from the exposed intracranial portion of the eighth nerve during vestibular nerve section. Electroencephalogr Clin Neurophysiol. 1994;92(4):316-20. doi: 10.1016/0168-5597(94)90099-x. PubMed PMID: 7517853.

16. Woo J, Miller CA, Abbas PJ. The dependence of auditory nerve rate adaptation on electric stimulus parameters, electrode position, and fiber diameter: a computer model study. J Assoc Res Otolaryngol. 2010;11(2):283-96. Epub 2009/12/22. doi: 10.1007/s10162-009-0199-2. PubMed PMID: 20033248; PubMed Central PMCID: PMCPMC2862915.

17. Woo J, Miller CA, Abbas PJ. Biophysical model of an auditory nerve fiber with a novel adaptation component. IEEE Trans Biomed Eng. 2009;56(9):2177-80. Epub 2009/06/02. doi: 10.1109/TBME.2009.2023978. PubMed PMID: 19497810.

18. Glowatzki E, Fuchs PA. Transmitter release at the hair cell ribbon synapse. Nat Neurosci. 2002;5(2):147-54. doi: 10.1038/nn796. PubMed PMID: 11802170.

19. Winter IM, Robertson D, Yates GK. Diversity of characteristic frequency rate-intensity

20. Smith RL, Zwislocki JJ. Short-term adaptation and incremental responses of single auditorynerve fibers. Biol Cybern. 1975;17(3):169-82. PubMed PMID: 1125344. 
651 22. Liberman MC. Auditory-nerve response from cats raised in a low-noise chamber. J Acoust

652 Soc Am. 1978;63(2):442-55. PubMed PMID: 670542.

653 23. Louage DH, van der Heijden M, Joris PX. Temporal properties of responses to broadband

654 noise in the auditory nerve. J Neurophysiol. 2004;91(5):2051-65. doi:

655 10.1152/jn.00816.2003. PubMed PMID: 15069097.

656 24. Bourien J, Tang Y, Batrel C, Huet A, Lenoir M, Ladrech S, et al. Contribution of auditory

657 nerve fibers to compound action potential of the auditory nerve. J Neurophysiol.

658 2014;112(5):1025-39. Epub 2014/05/21. doi: 10.1152/jn.00738.2013. PubMed PMID:

65924848461.

660 